\title{
Computed tomography angiography diagnosis of the accessory right coronary artery-pulmonary artery fistula: a case description
}

\author{
Pei-Qi Wu^, Fen-Ling Guo, Li-Wei Yu, Xiao-Ming Mao, Gao-Bo Wu \\ Department of Radiology, Southern University of Science and Technology Yantian Hospital (Shenzhen Yantian District People's Hospital), \\ Shenzhen, China
}

Correspondence to: Fen-Ling Guo. Department of Radiology, Southern University of Science and Technology Yantian Hospital (Shenzhen Yantian District People’s Hospital), Shenzhen, China. Email: fenglling@qq.com.

Submitted Nov 23, 2020. Accepted for publication Jan 20, 2021.

doi: $10.21037 /$ qims-20-1270

View this article at: http://dx.doi.org/10.21037/qims-20-1270

\section{Introduction}

The accessory coronary artery directly originates from the aortic sinus in addition to the left and right coronary arteries, so it is also called the third coronary artery (1). Coronarypulmonary fistula is a rare abnormal connection between coronary artery or its branches and pulmonary artery (2). The accessory coronary artery and pulmonary artery fistula occur at the same time is even rarer. Computed tomography angiography (CTA) is a reliable noninvasive diagnostic method in the diagnosis of coronary anatomy abnormalities. This article reports a rare case of the accessory right coronary artery-pulmonary artery fistula diagnosed by CTA.

\section{Case presentation}

A 52-year-old male patient visited our hospital because of activity induced chest tightness. His electrocardiograms and chest radiography were normal. The laboratory examinations, including troponins, were also normal. Then he underwent CTA on 28 August 2019 and it showed a supernumerary vessel origins from the right coronary sinus, known as the accessory right coronary artery (Figure 1A,B). The accessory right coronary artery travels tortuously, passing through the front of the main pulmonary artery in a "U" shape (Figure $1 C, D$ ), and finally it is injected into the pulmonary artery trunk through a small fistula (Figure 1E,F).

\section{Discussion}

Most of the accessory coronary arteries originate from the right coronary sinus, and a small portion originates from the left coronary sinus. Generally, the accessory coronary arteries do not cause hemodynamic changes, so they are considered to be benign anatomical abnormalities of heart (1). The accessory coronary artery provides collateral circulation and has an important compensatory effect for patients with severe stenosis or complete occlusion of the ipsilateral coronary artery (3). If the origin of the accessory coronary artery is very close to the opening of the ipsilateral coronary artery, the catheter may be mistakenly inserted into the accessory coronary artery during coronary angiography. In addition, accidental injury to the accessory coronary artery during cardiac surgery can cause massive bleeding. Coronary-pulmonary fistula is a rare congenital or acquired abnormal connection between the coronary artery or its branches and the pulmonary artery (2). Blood is shunted from the coronary artery to the pulmonary artery through the fistula. In patients with small fistulas, hemodynamic changes are too slight to cause clinical symptoms. It is worth noting that the diameter of the fistula of some patients will increase with age, and the blood shunt increases accordingly, which can cause symptoms in adulthood. When the amount of blood shunt from the fistula is large, coronary blood perfusion pressure would reduce rapidly

\footnotetext{
^ ORCID: 0000-0003-1329-0900.
} 

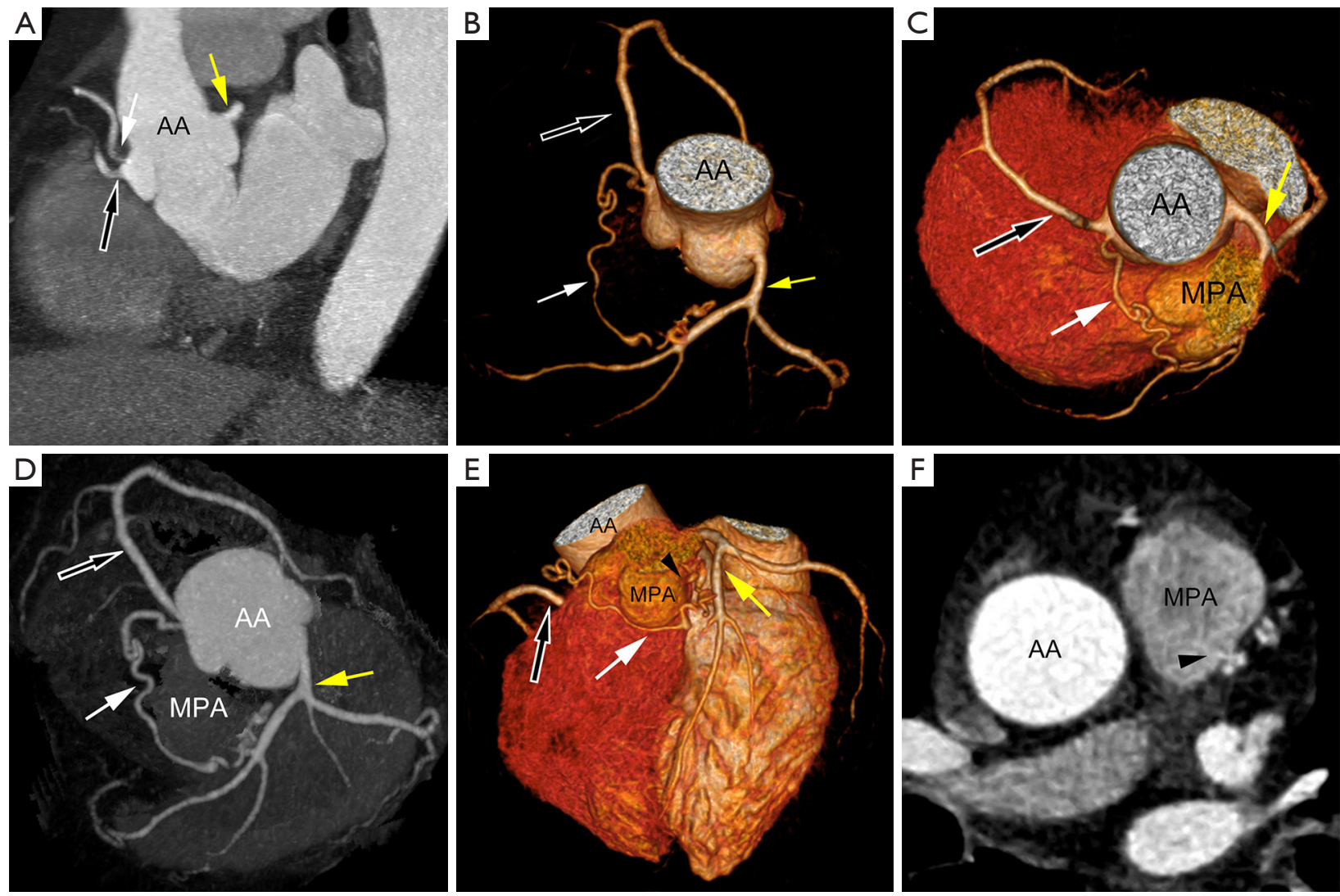

Figure 1 The accessory right coronary artery-pulmonary artery fistula. The reconstructed image shows that two blood vessels originate from the right coronary sinus of the AA (A,B,C,D). The MIP image shows that the opening of the right coronary artery (black arrow outlined by white) is located below the opening of the accessory right coronary artery (white arrow) (A,B,C,D). The left coronary artery normally opens in the left coronary sinus (yellow arrow) (A,D). The VR image shows the opening and the proximal segments of the right coronary artery (black arrow outlined by white), the accessory right coronary artery (white arrow), and the left coronary artery (yellow arrow) (C,D). The VR image shows that the accessory right coronary artery (white arrow) travels tortuously in front of the MPA in a U-shape (C,D). The normal right coronary artery (black arrow outlined by white) and left coronary artery (yellow arrow) can also be seen (A,B,C,D,E). The VR image shows that the end of the accessory right coronary artery is connected to MPA (black arrowhead). (E). Axial CT image shows that the contrast medium in the accessory right coronary artery is injected into the MPA through the small fistula (black arrowhead), which is named the "jet sign" (F). MIP, maximum intensity projection; AA, aorta; VR, volumetric reproduction; MPA, main pulmonary artery; CT, computed tomography.

during diastole, leading to myocardial ischemia and a series of symptoms. This phenomenon is also known as "coronary blood stealing (4)." Severe coronary pulmonary fistula can even lead to acute myocardial infarction, heart failure, malignant arrhythmia and sudden death (5). Therefore, the early and accurate diagnosis of coronary artery-pulmonary fistula has important clinical significance. The prevalence of congenital coronary artery anomalies detected by imaging modalities ranges from $0.21 \%$ to $5.79 \%$ (6). Compared with coronary angiography, CTA makes it possible to visualize coronary artery anatomy in a non-invasive way (7). The volume reconstruction technology of CTA can display the opening of the coronary artery and its full course in three dimension (3D) (8). The case reported in this article reflect the diagnostic value of CTA in diagnosing coronary anatomy abnormalities.

\section{Acknowledgments}

Funding: This work was supported by grants from the 
National Natural Science Foundation of China (Grant Number 81701662) and the Science and Technology Program of Shenzhen Yantian District (Grant Number YTWS20200204).

\section{Footnote}

Conflicts of Interest: All authors have completed the ICMJE uniform disclosure form (available at http://dx.doi. org/10.21037/qims-20-1270). The authors report grants from National Natural Science Foundation of China (Grant Number 81701662), grants from Science and Technology Program of Shenzhen Yantian District (Grant Number YTWS20200204), during the conduct of the study.

Ethical Statement: Written informed consent was obtained from the patient for publication in this case report and any accompanying images.

Open Access Statement: This is an Open Access article distributed in accordance with the Creative Commons Attribution-NonCommercial-NoDerivs 4.0 International License (CC BY-NC-ND 4.0), which permits the noncommercial replication and distribution of the article with the strict proviso that no changes or edits are made and the original work is properly cited (including links to both the formal publication through the relevant DOI and the license). See: https://creativecommons.org/licenses/by-nc-nd/4.0/.

\section{References}

1. Akkaya H, Gunturk EE. Coronary artery anomalies and dominance: data from 7,858 patients in a single center in Turkey. Minerva Cardioangiol 2020. doi: 10.23736/S0026-
4725.20.05279-2.

2. Graidis C, Dimitriadis D, Karasavvidis V, Dimitriadis G, Argyropoulou E, Economou F, George D, Antoniou A, Karakostas G. Prevalence and characteristics of coronary artery anomalies in an adult population undergoing multidetector-row computed tomography for the evaluation of coronary artery disease. BMC Cardiovasc Disord 2015;15:112.

3. Bigler MR, Seiler C. The Human Coronary Collateral Circulation, Its Extracardiac Anastomoses and Their Therapeutic Promotion. Int J Mol Sci 2019;20:3726.

4. Huang $Z$, Liu Z, Ye S. The role of the fractional flow reserve in the coronary steal phenomenon evaluation caused by the coronary-pulmonary fistulas: case report and review of the literature. J Cardiothorac Surg 2020;15:32.

5. Liu X, Zhang L, Qi Z, Fan M, Ge J. The characteristics of coronary-pulmonary artery fistulas and the effectivity of trans-catheter closure: a single center experience. J Thorac Dis 2019;11:2808-15.

6. Pérez-Pomares JM, de la Pompa JL, Franco D, Henderson D, Ho SY, Houyel L, Kelly RG, Sedmera D, Sheppard M, Sperling S, Thiene G, van den Hoff M, Basso C. Congenital coronary artery anomalies: a bridge from embryology to anatomy and pathophysiology--a position statement of the development, anatomy, and pathology ESC Working Group. Cardiovasc Res 2016;109:204-16.

7. Zhou K, Kong L, Wang Y, Li S, Song L, Wang Z, Wu W, Chen J, Wang Y, Jin Z. Coronary artery fistula in adults: evaluation with dual-source CT coronary angiography. $\mathrm{Br}$ J Radiol 2015;88:20140754.

8. Ali A, Colledge J, Sri I, Missouris C. CT: the imaging of choice in the diagnosis of coronary artery fistulae. BJR Case Rep 2016;2:20150492.
Cite this article as: Wu PQ, Guo FL, Yu LW, Mao XM, Wu GB. Computed tomography angiography diagnosis of the accessory right coronary artery-pulmonary artery fistula: a case description. Quant Imaging Med Surg 2021;11(10):4488-4490. doi: 10.21037/qims-20-1270 